\title{
Ovarian Luteinized Thecoma
}

National Cancer Institute

\section{Source}

National Cancer Institute. Ovarian Luteinized Thecoma. NCI Thesaurus. Code C4203.

A variant of ovarian thecoma characterized by the presence of lutein cells. It is associated with a lower frequency of estrogenic manifestations compared to typical thecomas. In a minority of cases androgenic manifestations are present. 